\section{Should There Be An Association of Hospital Epidemiologists?}

The Association for Practitioners in Infection Control (APIC) was formed in April 1972. Its membership on July 31,1980 was 5,713 , of whom about $85 \%$ were initially trained as nurses, $5 \%$ as microbiologists, $5 \%$ as physicians, and $5 \%$ in other fields. In 1978 Dr. John McGowan began a Hospital Epidemiologist's Newsletter. As of November 1979 the Newsletter was being circulated to 220 people, 206 of whom were physicians. The list has grown by about 85 since then. Informal meetings of this group were held at the 1978 and 1979 ICAAC meetings and at the Second International Conference on Nosocomial Infections in Atlanta in August 1980.

In the June 1980, Hospital Epidemiologist's Newsletter (its seventh issue) several people, including the author, advocated the formalizing of a Hospital Epidemiologists' Group (HEG). At the August meeting in Atlanta, Dr. Glen Mayhall presented a proposal to do this, identifying the elements of an organization and the decisions that would have to be made in the course of its establishment. A more detailed proposal probably will be made at ICAAC in September.

This editorial is a personal view, from the perspective of a hospital epidemiologist, of the pros and cons of forming an HEG. It incorporates the ideas of many others, expressed in conversations and commentaries at the Atlanta meeting. Assuring accuracy of quotation is difficult and individual acknowledgment awkward, leaving me able only to offer my grateful thanks.

One example of a specific function that might be done more effectively by an HEG than by APIC is to combat or modify inappropriate proposed legislation or regulatory agency policies. While I am fairly optimistic about possible proposals from the JCAH and the CDC (the recent hepatitis guidelines notwithstanding), these groups issue standards and guidelines only. The regulatory agencies are of greater concern. The EPA's recent proposals about ethylene oxide and infectious waste bode ill for the future. The FDA, OSHA, EPA and state agencies sometimes show little understanding of our views or interest in our opinions. APIC is attempting to develop consensus views on specific infection control practices, much in the style of AORN's operating room guidelines. This is an important and useful activity, but the function I envision would be more narrowly focused and reactive. A special mechanism within APIC would need to be developed to accomplish the quick response that may be needed.

A critical need for the field of hospital infection control is regular funding for research. At the recent CDC conference there were many calls for a better scientific basis for our policies, but it was not until the ending round table discussion that Dr. Edward Kass identified one of the major impediments. Within the federal government, the CDC is interested in hospital infections, but the NIH grants money for research. In spite of SENIC and the recent IV team contract, it doesn't appear likely that the CDC plans any largescale research funding activity. The NIH (which might be better titled the National Institutes of Biomedical Research) has shown little interest in hospital infection control research. There was, for instance, only one $\mathrm{NIH}$ representative and an assistant at the CDC conference. The NIH spends its billions largely on projects the results of which will stimulate other research, rather than "end stage," smaller scale research to answer immediately applicable questions. APIC has made initial overtures to the NIH, attempting to increase interest in infection control research. But the resolution of this "Catch-22" is going to require a rigorous, highly academic and persistent campaign, perhaps even extending to the congressional level.

I shall refer to these first two functions as the "pressure group" functions. Are there other groups that can perform them? The new Division of Nosocomial Infections of ASM may have this potential, and certainly will enable us to influence ASM. There should be ICAAC sessions on Nosocomial Infections in 1981, as there were in 1975-1979. ASM is also experienced at interacting with the government. It is a well-funded group whose professional staff is effective 
and respected in Washington. But the ASM is fundamentally an association of microbiologists. It is not even clear that hospital epidemiologists will comprise a majority of the new Division. Divisional opinions can be expressed only through the overall organization's Board of Public and Scientific Affairs, although that committee sometimes presents minority views.

The American Hospital Association (AHA) Advisory Committee on Infections Within Hospitals is likely to hold sound opinions (its chairman is Dr. Theodore Eickhoff), but it is not an elected body and must operate through the AHA. Heretofore its main function has been to advise hospitals on infection control matters, primarily via the manual Infection Control in the Hospital, now in its 4th edition. The AHA does have an effective lobbying mechanism, but the organization is concerned mainly with matters of interest to administrators-reimbursement, health care organization, regulations on cost containment-rather than clinical problems. Neither the Infectious Diseases Society of America nor the National Foundation for Infectious Diseases (NFID) seem likely forums for our interests. It is really only APIC whose interests are identical to ours. APIC's emphasis and priorities may be different, but its goals are very similar.

A more nebulous, albeit important, potential benefit of an HEG is a better sense of identify for hospital epidemiologists. For many in the academic world, the stature of infection control is low. (This seems a general fate of public health-oriented endeavors.) To enhance our standing (the "stature goal"), an HEG would have to accomplish a great deal, perhaps requiring more time than many of us have to contribute. National meetings, coordinated research, and an official journal would be important elements.

A final argument for an HEG is the need to establish a stable financial basis for the Newsletter. Currently it is supported by the NFID, but this is unlikely to continue for more than a few years.

If an HEG is to be formed, we must decide how simple or how complex the organization should be: incorporated or not, boards and committees or just a few officers, whether to embark on continuing education programs, coordinated research, independent national meetings, etc. I believe that a simple HEG can accomplish the pressure group function, while a "stature goal" would require a complex HEG. Those who favor a simple HEG should recognize that organizations tend to grow; keeping it simple may not be possible.

Before founding an HEG to advance these somewhat academic goals, we should realize that the organization's orientation may change radically in the next decade. Today the Newsletter mailing list membership is strongly weighted toward university hospitals and other teaching institutions. But the number of hospital epidemiologists may grow as much in the next decade as the number of infection control practitioners did in the last. In ten years the $\mathrm{HEG}$ could be much more interested in the needs of community hospitals, weakening the rationale for founding a separate group.

To me the thorniest questions about an HEG are those concerning criteria for membership. Possible criteria include: (1) a graduate degree (presumably including an M.S. and an M.P.H.), and (2) directorship of a hospital infection control program or membership in a state or federal health agency with an interest in hospital infection control. Unfortunately, these criteria would result in the inclusion of some physicians with peripheral interests in infection control while excluding some highly capable infection control practitioners who actually run infection control programs that are in theory run by physicians. Some of these infection control practitioners may be included by adding as a membership criterion first authorship of a publication dealing with infection control.

This discussion cannot be complete without an attempt to confront the problem of elitism. I am proud to be working in the field of health care that has had the most progress in the effort to reduce the hierarchical barriers between physicians, nurses, and others. Hospital epidemiologists should be certain to eliminate elitism as a motivation for forming an HEG. Yet, as invidious as elitism is, it is also a reality of the world in which we function. One would like to think that the merits of the argument would be determining, but the NIH or the EPA may be more likely to respond to a group of physicians than to a group perceived as being composed predominantly of nurses. This potential increased effectiveness may extend to achieving better physician adherence to recommended patient care procedures.

How would the formation of an HEG affect APIC? Many hospital epidemiologists who oppose an HEG are strongly motivated by the desire to avoid damaging APIC or creating resentment among non-hospital epidemiologist APIC members. Although there may have been some hesitation initially, APIC has now issued an explicit welcome to hospital epidemiologists. Infection control practitioners, for whom physician suport is a permanent problem, will be discouraged by the formation of an HEG, even though there are valid reasons for it.

Even more importantly, how would the formation of an HEG harm the nation's infection control effort? Energy would be diverted into duplicate administrative functions. While I doubt that APIC and an HEG would hold opposite views on any issue, fragmentation might dilute the message.

Can APIC accomplish the "pressure group" functions? Its leadership has indicated a willingness to explore the legalities of lobbying and to consider developing mechanisms to meet these needs. Can the hospital epidemiologists find an identity as a section within APIC? I would be unhappy if section status were extended as a special privilege to only one APIC 
subgroup. But there are other groups of members (e.g., those from VA, pediatric, or university hospitals) with valid needs for sections. Several APIC board members have indicated an interest in exploring this option. Its development would require thoughtful analysis; the possibilities and pitfalls are worth a separate editorial. Clearly, the majority of the APIC Board must remain infection control practitioners and the organization must serve first the needs of the bulk of its membership: the community hospital practitioners. But APIC may now have the self-confidence to use creatively the hospital epidemiologists' challenges.

At the ICAAC Hospital Epidemiologists' meeting, we should decide whether or not to proceed at this time with the formation of an HEG. Each of us must weigh the resultant fragmentation, practitioner discouragement, and administrative duplication against whether we can accomplish the pressure group and stature functions better with an HEG than within APIC or the ASM. This is the only view I would strongly advocate: I think it inadvisable to form an HEG out of a minority of the hospital epidemiologists. The consensus at the New Orleans meeting should be clearly in favor of an HEG or we should wait another year or two before reconsidering the issue.

Frank S. Rhame, M.D. Box 421

University of Minnesota Hospitals and Clinics Minneapolis, Minnesota 